\title{
Register rerum vol. 97 et 98
}

Die fett gedruckten Zahlen bedeuten indiquent des travaux originaux. - Bb. $=$

Buchbesprechungen - E

A

Abduzensparese mit Exophthalmus

bei Basedow 97, 268 Ablatio retinae 97, 121, 326; 98, 1

Vererbung der - 97, 235

traumatische 98,91

durch indirektes Trauma 98, 183

Operation der - 98, 363 Akkommodation 97, 323 (Rev.)

- $\quad$ Effects of Lenses and Convergence upon - 97, 328

Acetijlcholíne 98, 250

Acide chronique, Brûlures de Гæil par - 97, 395

Acné Tosacée et Iritis 98, 242

Adaptation, Dependency of the Contrast-Sensitivity of the Eye upon

- 97, 289

Adenoma sebaceum 97, 234

Adiesch.es Syndrom 97, 222

Agonie et la mort de quelques maladies oculaires 97, 392

Aiguiseurs, Kératopathie profession-nelle des - 97, 351

Albinisme 97, 209

complet 97, 124 Aliergie 97, 389

Vererbung der - 97, 242 Altersevscheinungen am Auge 97, 238 Amblyopia ex anopsia 98, 162

$\Lambda \mathrm{mbl} \mid$ opie, transitorische - nach

der Staroperation 98, 300 Ametrope, Sehen von 98, 56 Amyloideinlagerungen in der Kon-

junktiva 97, 53 Anaphylaxie 97, 359 Angiomatosis haemorrhagica Iiereditaría 97, 233

- $\quad$ und Kleinhirnangiom 98, 170Aniridie congénitale 98, 221 Anophthalmus, vorgetäuschter

98, 100Anopsia, Amblyopia ex a. 98, 162Anterior Capsule 97, 325Aphakia, congenital - with

Hyaloid

Artery and Retinal Fold 97, 79 Aqueous humour, formation of the

- $\quad 97,397$

Arterien, Veränderungen der - in der Retina und in andern Orga-nen 98, 176

Astigmatismus, allgemeine Störungen bei - 98, 375

iginalarbeiten. - Les chiffres gras ick ciphers signify original papers. í Notices - Livres

nouveaux.

Atrophia gyrata chorioideae et retinae

98, 362* Augenabweichungen, Kombination

angebo ener 98, 364 Augendruck, gleichzeitige Einwirkung

einiger Miotika und Mydriatika

auf den - 98, 16 Augenmuskeln, erbliche Anomalien 
der - 97, 204 - zentrogene Einflüsse auf die -

98, 164 Autokinetische Bewegungsempfindun-

gen 97, 263 Avitaminosen 97, 48

B

Basedowsche Krankheit mit Exophthalmus und Abduzensparese

97, 268, 98, 251Beleuchtung, Abhängigkeit der Seh-

schärfe von der - 97, 322

- $\quad$ als Unfallsursache 98, 112Beleuchtungsintensität, Einfluß der -

auf die Größenmessung des blin-

den Flecks 97, 364 Benzol, traitement du trachome par

le - 97, 390 Berufskrankheiten 97, 388, 98, 106

(Rev.), 378 (Bb.) Besnier-Bæck-Schaumann, Maladie

de - 98, 230 Bewegungsempfindungen, autokinetische 97, 263 Bíologie in der Augenheilkunde

97 ,

128 Biomicroscope et diaphanoscopie 97,

391 Blendung 98, 90

- $\quad$ Schädigung der Makula durchSonnenstich 98, 173

Blennorrhoea adultorum 97, 267 Blepharophímosis 97, 204 Blindenwesen 98, 117 (Rev.)

Blinder Fleck, Einfluß der Beleuchtungsintensität auf die Größenmessung des - 97, 364

Blindheit, erbliche 97, 244

- $\quad$ das Antlitz der - in der Antike

98, 380 (Bb.)

- $\quad$ in Griechenland 97, 90Blindness in Australia 98, 249

JJ Register rerum

Blind spot, visibility of the -98 ,

191 (Bb.) Blitzschlag, Verletzungen durch -

98, 90 Blood supply of the Optic nerve and

Retina 97, 328 Blutgruppen, Erblichkeit der - 97,

244 Brucellose, complications uvéales de

la - 98, 239 Brûlures de Væil par Гacide chro-

mique 97, 395 Bulbus, Evisceratio b. mit Abtragung

des Skleralsackes 97, 26

C

Capillaropathia retinalis 98, 129 Cataracte polaire antérieure micro-

scopique 97, 125 Catarrhe printanier en Grèce 97,

340 (Bb.) Chiasma, Tumoren des - 98, 167 Chinin-Bisulfat bei Trachom 97, 113 Choc,

medications de choc en oph-

talmologie 97, 131 (Sb.) Cholestérine dans les lames de la

cornée 97, 390 Choñoidea, Atrophia gyrata der -

98,362

Décollement de la-98, 344

Tumeurs de la - 98, 346

Sarcome plat de la - 97, 394 Chorioideremie 97, 232 ChoГioiditis 98, 334

Ch. familiale 98, 224 Chorioretinitis, Jensensche 97, 261 Conjunctiva 97, 28 (Rev.), 96 (Rev.)

Konjunktivitis, Bewertung des bak-

teriologischen Befundes bei - 97, 20, 28, 76 
- $\quad$ C. printanière et lumière 97,389

- $\quad$ C. gonorrhoica, Uliron bei - 97,341

Contrast-Sensitívíty, Dependency of C.

of the Eye upon Adaptation 97,

289 Convergence, Effect of - upon the

Accommodation 97, 328 Corectopie 98, 221 Corépraxie dans un cas d'occlusion

pupillaire 97, 122 Cornea, Erblichkeit bei Affektionen

der - 97, 217 (Rev.)

Sensibilität der - in verschie-denen Lebensaltern 98, 189

Cholestérine dans les lames de la-97, 390

vol. 97 et 98

Cornea guttata bei chronischer Irido-zyklitis 98, 178

C. guttata und Keratokonus 97, 266

fleckige Dystrophie der - 98, 311

punktförmige und ringförmige Dystrophie der - 97, 374, 375

Corps étrangers de la $-97,333$

Kyste épithéliale pigmentaire de la - 97, 123

- $\quad$ Greffes cornéennes 98, 164Corps étrangers de la cornée 97, 333Corps vitré, Scintillement blanc du -

98, 187 Corpus ciliare, Diathermie des - bei

Glaukom 98, 178. Cristallin en survie 97, 159

- $\quad$ Métabolisme hydrocarboné du $-98,142$

Crossing over bezüglich des X-Chro-

mosoms 97, 227. Crouzon-Maladie 98, 305

$\mathrm{D}$

Dakryozystorhinoslomie 98, 362 Déchirure conjonctivale par la projection du contenu d'une balle de golf 97, 124 Déchirure rétinienne 97, 121 Décollement de la rétine 98, 1, 2 DefizienzNeuritis 97, 265 Degeneratio tapeto-chorioidealis cen-

tralis progressiva 98, 6 Degeneration der Konjunktiva 97, 52 Dents et le nerf optique 97, 133

Descemetsche Membran, ringförmige

Zirkulärleiste der - 98, 159 Detachment of the retina 97, 326 Detachment of the Retina 97, 326

Entstehung und Rückbildung der Katarakt bei - 98, 190

Complications uvéelles du Diabète 98, 244

Diaphanoscopie et Biomicroscope 97,

391 Diathermie 97, 327, 328, 329

- D.-Stichelung des Corpus ciliarebei Glaukom 98, 178

Dinitrokresol, Erzeugung einer Katarakt durch - 98, 171 Disc, Pallor of the - 97, 326

Dynamometrie 97, 331 Dijsostose cranio-faciale 97, 386, 98,

' 305 Dysostosis multiplex 97, 220

Register rerum

vol. 97 et 98

III

E

Eclampsie 98, 177

Ectopia lentis, Vererbung der - 97, 
207 Ectopic-Zonula 98, 149 EctГopion de I'uvée 98, 220 Einäugigkeit, das Risiko der - 98, 364 Einschlußblennorrhoe 97, 31 Eisensplitterverletzungen 98, 75 Elektrischer Strom, Augenstö $\pi$ ingen duГch den - 97, 266, 98, 90 Emmetropisierung 97, 213 Enophthalmus tzaumatícus 98, 169 Entoptiques phénomènes et la rétine 98, 191 (Rb.) Ent Гopion, Rehandlung des - 97, 116

- $\quad$ verei $\pi$ fachte Tarsoplastik gegenNarben - Eine 97, 69

Enucleation, Effect of - of one eye

on the Growth of the Face 97, 329 Epithéliomas du limbe 97, 391 Esérine, le diagnostic différentiel entre le pseudo-tabès et le tabes par - 97, 122 Eugenik 97, 244 Evisceratio bulbi mit Abtragung des Skleralsackes 97, 26 Excitation vestibulaire et la T. A. R. 97, 127 Exophtalmie unilatérale 97, 334; 98, 124 Exophthalmus 97, 376 (Rev.) mit Abduzensparese und Rasedow 97, 268 and Graves' disease 98, 251 pulsierender mit Glaukom 98, 100 $\mathrm{F}$ Farbenblindheit 97, 226 ff. Filaria in der Konjunktiva 97, 34 Fleischerscher Ring, familiäres Auftreten des - 97, 240 Fluid Layer before the Eye 98, 284 Follikulose und Trachom 97, 105 FosterKennedy-Syndrome 97, 397 Frühjahrskatarrh 97, 50, 340 (Rb.), 389 Fundus in the Diabetic 97, 326 Funktionelle Störungen 98, 368 Fuseau de Krukenberg 97, 125

G

Galaktose und Katarakt 97, 326, 95,

361 Ganglion sphenopalatinum 97. 381

Gargoylismus 97, 220

Gasbrand 98, 95

Gehimsklerose, Netzhauttumoren bei tuberöser - 97, 234

Gehimtumoren, Redeutung d. Augen-symptome fur die topische Diagnose der-97, 129

Gelbkreuzerkrankungen der Augen 97, 271

Glaskörper, erbliche Affektionen des - 97, 226

- $\quad$ rezidivierende Rlutungen des -

97, 235

-匹 Reziehungen des - zur Retina

98, $\quad 188$

Glaukom, Redeutung des Wortes - im Altertum 98, 172

Heredity of - 97, 211, 98, 43

G. phakogeneticum 97,267

G. et naevus flammeus - 97, 334 - Reeinflussung der Transsudation

aus den Netzhautgefäßen durch ein - 97, 146

Diathermiestichelung des Corpus ciliare bei - 98, 178

G. secondaire, la transfixion de Tiris dans le $-97,393$ 
Gliom des Optikus - 97, 268 Gíioma retinae, Vererbung des - 97,

212 Glycosu

globe oculaire 97, 334 Gonokokken-Iritis 98, 240 Grains de plomb intraoculaires 97,

394 Graves' disease 98, 251 Greffe cornéenne 97, 121, 123, 98, 364 Gunnsches Phänomen 97,

205

II

Haemangioma orbitae 97, 268 Hämophilie, Vererbung der - 97, 244 Haemorrhage experimental - into

the eye 98, 253 Harada - Maladie 98, 339 Heerfordt-Syndrome 98, 230 Hémorragies

récidivantes, origine

tuberculeuse des - 97, 396 Hereditary Eye Disease 97, 325 Herpes zoster und sympathische

Ophthalmie 98, 367 Heterochromie 98, 327 Hétérophorie 97, 120 Hippel-Lindausche

Erkrankung 97,

233, 98, 170 van der Hoevesches Syndrom 97, 221 Hyaloid artery 97, 99 Hyperphorie altérante 97, 120

jy Register rexim vol. 97 et 98

Hypertension 98, 343

experimentelle 97, 273

artérielle rétinienne 97, 126 Hypophyse et pression vasculaire

rétinienne 97, 66

- $\quad$ Störungen der - durc!ı Sella-fraktur 98, 102

Hypopyon, Iritis récidivante à -98 ,

237 Hypotony after Scleroc, orneal

Trephining 97, 326

i

Idiotic infantile aniarautische 97, 229 Indische Erfahrungen 97, 261 Infection focale 98, 235

Iontophoresis 97, 166 Iridectomie transsclérale 97, 124 Irídencleisis Holth 97, 327 Iridocyclites 98, 225 (Rev.), 328

Cornea guttata bei chronischer $-98,178$

und Poliosis 97, 265

Iris, erhliche Affektionen der - 97,

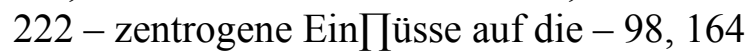

Culture de tissu irien in vitro 98, 217

Neubildungen der - 97, 302

Transfixion de - dans le glau-coiПe secondaire 97, 393

Iriskolobom, Operation einer senilen Katarakt bei” angeborenein - 97, 270

Iritis 98, 225 (Rev.), 328

$\mathrm{J}$

Jensensche Chorioretinitis 97, 261

$\mathrm{K}$

Kammerwinkelkontaklglas 97, 330 Katarakt, Vererbung der - 97, 222, 98, 169

Erzeugung einer - durclı Dinitro-kresol 98, 171

Lactose- und Galactose-K. 97, 328, 98, 361

Rosetten-K. 97, 266

Ultrastrahlen-K. 98, 168

Cat. polaire antérieure micro-scopique 97, 125 
bei einheimischer Sprue 98, 291

Entstehung und Rückbildung des Zuckerstars 98, 190

Kataraktoperation 97, 392, 393, 98, 254, 367

intrakapsuläre 98, 363, 371

bei angeborenem Iriskolobom 97, 270

transitorische Amblyopie nach der - 98, 300

Keratitis der Lackarbeite $\Gamma$ 98, 166 Keratitis bullosa 98, 252

posltraumatica 98,160

profunda 98,365

Vermeidung der - punctata 98,

81 Keratoconjunctívitis sicca 97, 47

- $\quad$ mit Arthritis 97, 267Keratokonus und Cornea guttata 97,

266 Keratopathie professionnelle des

marbriers et des aiguiseurs 97 ,

351 Keratoplastik 98, 365 Kleinhirnangiom und Angiomatosis

retinae 98, 170 Koch-Weeks-Epídemie 97, 34 Kohlensäure-Anhydrase der Linse 98,

361 Kolobome, Vererbung der - 97, 206 Kontaktglãser, aus Harzprodukten

hergestellte $-97,262$

- $\quad$ Kammerwinkel-K. 97, 330Knochenbildung, metaplastische - bei

Transplantation von Ureter in die

Vorderkammer 97, 284 Krukenberg, Fuseau de - 97, 125 Kupfer in der Linse 98, 366 Kyste

épithéliale pigmentaire de la

cornée 97,123

L

Lactose Cataract 97, 328 Laurence-Moon-Bardet-Biedlsches

Syndrom 97, 231 Lenses, Effect of - upon the

Accommodation 97, 328 Lèpre, Complications uvéales de la

- $\quad 98,242$

Lichen planus der Konjunktiva 97,

46 Licht, Zerstreuung von - durch die

Linse 97, 269 Lidplastik 97, 312 Linse, erbliche Affektionen der -

97, 222

- $\quad$ Zerstreuung von Licht durch die

- $\quad$ 97, 269

Kupfer in der - 98, 366 Lipodermoide 98, 366 Lipoidosen 97, 229 Lymphogranulomatosc der

Konjunktiva 97, 37

bénigne 98, 230 Lymphome des Rulbus 97, 59

VI Register $\Gamma \beta$ rum vol. 97 et 98

Retina of Australian Reptiles and Mammals 98, 253

Blood supply of the $-97,328$

Beziehungen de $\Gamma$ - zum Glas-körper 98, 188

Metabolismus der abgelösten - 97, 270

Capillaropathia retinalis 98, 129

DéchiruГe ret. 97, 121

Verletzungen der - 98, 103 
- $\quad$ Tumoren der - 97, 234Retinagefäße, Druck in den - 98,

102

- $\quad$ Beeinflussung der Transsudationaus den - durch ein Glaukom

97, $\quad 146$

- $\quad$ Verhalten der Arterieı andererOrgane bei Veränderungen der -

98, 176Retinal Fold 97, 79

Rétine et phénomènes cntoptiques 98, $191(\mathrm{Bb}$.)

- $\quad$ plane ciliaire, Malformation con-génitale de la - 97, 393

Retinitis centralis serosa 97, 330 Retinitis pigmentosa 97, 123, 230, .95,

253 Retino-Blastoma 98, 193 Rhumatisme 98, 233 Rickettsies et Trachome 97, 277

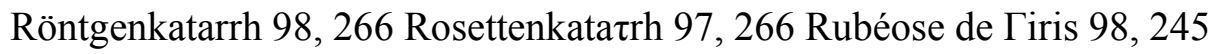

$\mathrm{S}$

Sarkom der Orbita 97, 268 Schådel, Anomalien des - 97, 239 Schüller-Christiansche Erkrankung

97, 385Schußverletzungen 98, 82Scintillement blanc du corps vitré 98,

187 Sclerocorneal Trephining, Hypotony

after - 97, 326 Scléropérikératite 98, 229 Sehschårfe 97, 322 (Rev.) Sehschwachenschulen 98,

123 Silbertherapie, Problematik der -

98, 51

Sklera, Erblichkeit bei Affektionen

der - 97, 217 (Rev.) Sklerose, multiple 97, 242 Sonnenstich, Schädigung der Macula

durch - 98, 173 Spaltlampenphotographie und -

-photometrie 98, 257 Spirochaetosis arthritica 97, 46 SpiГochétose ictéro-hémorragique

98, 238

Sprue, Katarakt bei einheimischer -

98, 291 Staphyloma congenitum 97, 265 Staphylome postérieur 97, 123 Strabismus,

Kongenitaler 98, 162

- $\quad$ Korrektur des paralytischen -bei Kontraktur der Antagonisten98, 162

- $\quad$ Traitement opératoire du - 97, 81 Sulfanilamid 97, 331

- $\quad$ Behandlung des Trachoms mit -98, 208

Sulfanilamido-Pyridine in the Ocular

Fluids of Rabbits 97, 356 Syphilis 98, 232, 334 - der Konjunktiva 97, 35

$\mathrm{T}$

Tabakrauch 98, 378 (Bb.)

Tabes, Diagnostic différentiel entre

le - et le Pseudotabès par

Esérine 97, $122 \mathrm{Ta}$ Soplastik, vereinfachte - gegen

Narbenentropium 97, 69 T.A. R. et excitation vestibulaire 97 ,

127 Temperature oculaíre 97, 334 Tobacco-Amblyopia 97, 329 Trachom 97, 96 (Rev.)

et Rickettsies 97, 277

Chemotherapie des - 98, 321

Behandlung des - mit Sulfanilamid 98, 208

Traitement du - par le benzol

$97, \quad 390$

Tränenröhrchen, Elongation der -

$98, \quad 366$

Tränenwege und Trachom 97, 116 Transfixion de Гiris dans le glau- 
come secondaire 97, 393 Transplantation von Konjunktiva 97,

62

Corneal-T. 98, 253

Früh-T. nach Denig bei Verätzun-gen 97, 329

metaplastische Knochenbildung bei - von Ureter in die Vorder-kammer 97, 284

Trichiasis, Behandlung der - 97, 116 Tuberculose oculaíre 97, 39, 396, 98,

227, 334 Tuberkulose, atypische 97, 338

- $\quad$ Uveitis tub. bei Zwillings-schwestern 98, 366

Tularämie 97, 39

Tumeurs de l'iris et du corps ciliaire 97, 124

de la choroïde 98,346

intraoculaires 98, 271

Register rerum vol. 97 et 98

$\mathrm{M}$

Macula lutca.

histologischer Befund bei Pseudo-tumor der - 98, 182

infantile Degeneration der - 97, 230

Schädigung der - durch Sonnen-blendung 98, 173

Magnet, Haabscher 98, 169 Maladies professionnelles 97, 388, 98,

106 (Rev.), 378 (Bb.) Marbric $\Gamma$ S, Keratopathie profession -

nelle des - 97, 351 Marfan-Syndrome 98, 201

- $\quad$ Vererbung des - 97, 207Membrane pupillaire, ïestes de - 98,

220 Mercurochrom 97, 31 Messungen am lebenden mensch-

lichen Auge 98, 370 Metabolisme hydrocarboné du

cristallin 98, 142 Metabolismus der abgelösten Netz-

haut 97, 270 Miosis congenita 97, 266 Miotika, gleichzeitige Einwirkung

einiger M. und Mydriatika auf

den Augendruck 98, 16 Mißbildungen 97, 243 Mongolismus, 97, 225 Muskelatrophie, progressive 97, 241 Myasthenie 97, 241 Mydriatika, gleichzeitige Einwirkung einiger Miotika und M. auf den

Augendruck 98, 16 Myopic. 97, 215, 98, 344

$\mathrm{N}$

Nachbilder 97, 400 (Bb.)

Naevus flammeus et glaucome 97 ,

334 Narbenentropium, vereinfachte Tarso-

plastik gegen - 97, 69 Nase und Auge 97, 380 N ebenhöhlenverschleierung , planigra-

phischer Nachweis einer -97 ,

346 Nerf optique et les dents 97, 133 Nervus opticus, erbliche Affektionen

des $-97,236$

offene Optikus-Röhre 97, 267

Zyste des - 97, 267 Neuritis, Defizienz-N. 97, 265 Neuritis Гetrobulbaris 97, 268

rhinogene 97, 380 Neuroblastoma in Twins 98, 253 Nystagmus, Vererbung des - 97, 206

der Bergarbeiter 98, 107

$\mathrm{O}$

Ocular tension, Treatment of Increased - by Diathermie 97, 328

One eyed worker 97,328 
Ophthalmia neonatorum, Bakteriolo-gie der - 97, 28

Ophthalmia sympathica 98,340

- traumatische 98,97

Ophtalmie métastatique 98, 337

Optic-Nerve, Blood supply of the $-97,328$

Optico-Encephalo-Myelitis 97, 268

Orbita 97, 382 (Rev.)

Orbitalplastik 97, 266

Orbital-Tumors 97, 382, 395, 98, 251, 253, 331, 365

Ossification intraoculaire 98, 95, 340

$\mathrm{P}$

Pallor of the disc 97, 326

Pannus, operative Behandlung des -

97, 115Papillenkonkremente 97, 268Parinaudsche Konjunktívitis 97, 36Pemphigus 97, 46, 391, 98, 367Periphlebitis retinae 97, 235Perspektive 97, 1

Phlyktäne 97, 41

Pigmentierung, Anomalien der - .97,

209 Planigraphischer Nachweis einer Ne-

benhöhlenverschleierung 97, 346 Plasmome 97, 54 Poliosis 97, 265 Pression vasculaire

retinienne et

Hvpophyse 97, 66 Proptosis 98, 252 Psammom der Orbita 98, 365 Pseudosklerose 97, 240

Pseudotumoren der Orbita 97, 382 - der Macula, histologischer Befund

bei - 98, 182 Psychologisches in der Augeiipraxis

98, 34Pterygium 97, 54

Pupille, erbliche Affektionen der - 97, 222

R

Recklinghausensche Krankheit 97,

203 Refraktion 97. 212 (Rev.), 317 (Rev.),

3. 327 Rendu-Osle $\Gamma$ sche Krankheit 97

233 Retina

erbliche Affektionen der -

97

226 